\title{
Looking back 10 years- Comparing aqueous cream with a beeswax and herbal oil cream in the provision of relief from post burn pruritis
}

\author{
Kay Wright* \\ Royal Brisbane and Women's Hospital, Australia
}

\begin{abstract}
A randomised controlled pilot study undertaken in a Burns Unit in a Large Metropolitan Australian Hospital in 2008 is the subject of this short discussion/ commentary on the study. Along with this short discussion of the pilot study, is a discussion on a commentary on the original article written in 2013 , in which the limitations of the study are examined. While some explanations are given for choices in the study and the subsequent article there is no in-depth discussion of those issues. A larger study was commenced after the Pilot Study but was not completed.
\end{abstract}

In 2008, while working as a clinical Nurse in a Major Adult burn Centre, I was involved in a Randomized Controlled Pilot Study, comparing Aqueous Cream with a Beeswax and Herbal Oil cream in the provision of relief from post burn pruritis. Patients on the unit had reported various intensity and occurrence of itch. Itch being the sensation inducing the urge to scratch [1]. A couple of the outpatients reported anecdotally in 2007-2008 that a cream they had sourced, was providing some relief from itch. Subsequent literature searches indicated that approximately $87 \%$ of burns patients experienced some itch post burn [2]. A pilot study was suggested to determine if the cream was effective. Over the course of the setup of the Pilot study it was decided that the patients would not be informed of which cream was to be applied. The creams were instilled into identical containers, but the appearance of the creams was different (white vs yellow). 78 patients on the adult burn Unit were approached to participate in the study. 52 (84\% male) participants were enrolled, 22 declined participation and 4 participants were excluded because they had a mild reaction when a patch test of the beeswax/herbal cream was applied. Patients arriving on the ward from ICU and HDU were excluded. Participants were randomly assigned to each group. An independent researcher used a random generator program to assign participants. 14 days of data collection occurred, using a visual analogue Scale [2] including, efficacy as well as application time, time to reoccurrence, antihistamine use and sleep disturbances.

The data indicated that within a Twenty-four-hour period, there were fewer applications of the beeswax/herbal oil cream and reduced use of antihistamines in that group. Participants also indicated that, itch was reduced $p=0.001$ [3], that itch reoccurred later $p \geq 0.001$ [3]. The outcome of the small pilot study indicated that a larger study was justified. A subsequent study was commenced but was not completed. I cannot comment on that study as I am unaware of the details. During the period immediately following the pilot study the use of the cream was high and approximately all patients on the unit used the cream. Due to restrictions in funding, the cream's use became dependent on the patients purchasing it themselves and use dropped off for some time. A change in funding has recently seen the cream being offered to patients again at no cost and use has slowly increased.

In 2013, Dr Savovic [4] in a commentary, analyses the pilot study. The article states, effect estimates, and confidence intervals were not reported. The statistics for the pilot study were provided by a statistician who was sent the data gathered and had no other contact with the researchers undertaking the study. Dr Savovic' stated that writing the paper using CONSORT guidelines [5] would have improved the information given in the pilot study report. Issues with the effectiveness of the blinding process for participants is discussed and as noted above, the difference in the creams was obvious and it would be feasible to assume that the staff applying the creams were aware of which cream was being applied. But as the decision was to blind the participants not the staff applying the cream this was determined not to be a problem. Whether the participants were aware of which cream was being applied is unknown as that was never discussed. The choice of Aqueous cream as the "Control" as also of concern to Dr Savovic'. At the time of the Pilot Study, the new beeswax/herbal cream was compared to the units then choice of moisturiser; Aqueous Cream. Peter Lewis in his reply to Dr Savovic' in the same journal, stated that, Aqueous Cream was consistent with the then current moisturising treatment in the literature. No research into the effectiveness or effect of Aqueous Cream was undertaken during the Pilot study. The concerns and limitations Savovic' [5] discuss in this article, related to the blinding process, how the random allocations were achieved as well as the choice of Aqueous Cream as comparison with the Beeswax/Herbal oil cream are valid to an extent, but limitations placed by the publisher of the article precluded

${ }^{\star}$ Correspondence to: Kay Wright, Clinical Nurse, Royal Brisbane and Women's Hospital, Australia, E-mail: Kay.Wright@health.qld.gov.au

Key words: beeswax/herbal cream, aqueous cream, itch, pruritis, randomised controlled pilot study, burns

Received: October 20, 2018; Accepted: November 01, 2018; Published: November 05, 2018 
that information being contained in the article. It was expected that during the larger study that many of the limitations occurring in the Pilot Study would be addressed, but unfortunately, the project was incomplete.

Anecdotal report from inpatients and outpatients who are continuing to use the cream would indicate further areas of study related to wound appearance as well as the effect on itch. Occupational therapists report that the use of the cream may have a detrimental effect on pressure garments, as they need to be replaced more often, and believe that one or more of the ingredients cause deterioration of the cloth in the garments. Personally, I like to use the beeswax/herbal cream in preference to other moisturisers on the unit. The application is smooth, the cream is oily but soothing on the skin and an application lasts for quite some time without the skin becoming dry and flaky. The process of examining the creams was interesting, a learning experience, one I had never been as closely associated with before or since. The team associated with this project was very supportive of each other and made the process from data collection to writing the article an effective learning experience.

\section{References}

1. Brooks JP, Malic C, Judkins LC (2008) Scratching the Surface - managing the itch associated with burns. A review of current knowledge. Burns 34: 751-760. [Crossref]

2. Field T, Peck M, Hemandez-Reif M, Krugman S, Burman I, et al. (2000) Post Burn itching pain, and psychological symptoms are reduced with massage therapy. $J$ burn care \& Rehabilitation 21: 189-193. [Crossref]

3. Lewis P, Wright K, Webster A, Steer M, Doubrovosky A, Gardner GE (2012) A Randomized Controlled Pilot study comparing Aqueous cream with a Beeswax and Herbal oil cream in the provision of relief from post burn pruritic. J Burn Care Res 33: 195-200. [Crossref]

4. Davodic' J (2013) A Pilot randomised trial of Medilixir cream shows some promising results, but control treatment may have been inappropriate. Focus on Alternative and complementary therapies 18: 105-106.

5. Schulz KF, Altman DG, Moher D, CONSORT group (2010) CONSORT 2010 Statement: Updated guidelines for reporting parallel group randomised trials. $B M J$ 240: c332. [Crossref]

Copyright: (C2018 Wright K. This is an open-access article distributed under the terms of the Creative Commons Attribution License, which permits unrestricted use, distribution, and reproduction in any medium, provided the original author and source are credited. 